\title{
A CPH-like picture in two patients with an orbitocavernous sinus syndrome
}

\author{
S Foerderreuther, R v Maydell, A Straube \\ Department of Neurology, Klinikum Grosshadern, Ludwig-Maximilians-University, Munich, Germany
}

\section{Cephalalgia}

\begin{abstract}
Foerderreuther S, Maydell R v, Straube A. A CPH-like picture in two patients with an orbitocavernous sinus syndrome. Cephalalgia 1997;17:608-11. Oslo. ISSN 0333-1024

Two patients with retroorbital pain syndromes with or without paresis of cranial nerves developed weeks after ipsilateral headache resembling chronic paroxysmal hemicrania $(\mathrm{CPH})$ but without autonomic features. These findings might support the hypothesis that $\mathrm{CPH}$ may be caused by a pathological process in the region of the cavernous sinus, as has been proposed for the Tolosa-Hunt syndrome (THS). $\square$ Chronic paroxysmal hemicrania, inflammation, orbital pain syndrome, orbitocavernous sinus syndrome'
\end{abstract}

Andreas Straube, Department of Neurology, Klinikum Grosshadern, Marchioninistr. 15, 81377 Munich, Germany. Fax.+49-(0)89-7095-3677, e-mail: u7×31av@sunmail.Irz.muenchen.de. Received 25 October 1996, accepted 31 December 1996

Chronic paroxysmal hemicrania (CPH), first described by Sjaastad and Dale in 1974 (1), is defined by the International Headache Society (IHS) as unilateral attacks of a severe orbital, supraorbital or temporal pain of 2 to $45 \mathrm{~min}$ duration occurring several times a day. Also, CPH shows signs of ipsilateral autonomic dysfunction, demonstrates a female preponderance and responds promptly to indomethacin treatment (2). The Tolosa-Hunt syndrome (THS) is characterized by episodes (days or weeks) of orbital pain associated with paralysis of one or more of the oculomotor nerves, sometimes accompanied by ipsilateral involvement of the optic nerve, the first trigeminal branch, and the sympathetic innervation of the pupil. This condition generally responds to corticosteroid therapy, but can take a relapsing and remitting course. In the past years we have seen two patients who initially had symptoms suggesting THS but who later developed retroorbital pain attacks resembling $\mathrm{CPH}$, as defined by the IHS and without the typical autonomic features.

\section{Case reports}

\section{Case 1}

A 31-year-old, previously healthy male first presented with a 2 months' history of constant bioccipital headaches that later changed into bilateral frontal pressing pain. He was admitted to the neurological department because of acute right frontotemporal

$\mathrm{CPH}=$ chronic paroxysmal hemicrania; THS=Tolosa-Hunt syndrome; $\mathrm{CH}=$ cluster headache; SUNCT=short-lasting, unilateral, neuralgiform headache syndrome with conjunctival injection and tearing; IHS=International Headache Society. and retroorbital stabbing pain with horizontal diplopia on right gaze, accompanied by numbness around the right nostril and below the right lower eyelid, slight dizziness and nausea. The following day he noticed diagonal diplopia.

Examination revealed an incomplete right abducens palsy, a slight paresis of the right inferior oculomotor division, and a discrete right trochlear palsy. There was no ptosis or pupillary abnormality. Facial sensation was diminished only in the distribution of the second trigeminal division; the corneal reflexes were equally brisk. Computed Tomography (CT) and contrast enhanced MRI with special slices of the sinus cavernosus and the hypophysis were normal, as were electroencephalography and haematological analysis including ESR, blood glucose, thyroid gland and vasculitis parameters (T3, T4, TSH, C-reactive protein, ANA, ANCA, C3, C4, Angiotensin converting enzyme). Magnetic resonance angiography showed a hypoplastic right transversus sinus, but no abnormalities in the region of the cavernous sinus. There was slight CSF pleocytosis (cells $13 / \mathrm{mm}^{3}$ ) with normal CSF protein and no oligoclonal bands. Since THS was suspected, steroid therapy was initiated. The headache and hypesthesia ceased after 2 days of treatment with $80 \mathrm{mg}$ oral methylprednisolone. The diplopia completely resolved over a period of 4 weeks.

Four weeks after tapering the steroids (administered for 8 weeks) the patient developed paroxysmal strictly right-sided temporal excruciating, knifelike headaches with a frequency fluctuating between 3 and 15 attacks in $24 \mathrm{~h}$, each one lasting $5-30 \mathrm{~min}$. Two weeks later the patient additionally noticed a steady pressing bilateral retroorbital pain, whichthough less intense-resembled the one he had 
experienced on admission and which resolved after a self-administered 4-day course of methylprednisolone $20 \mathrm{mg}$ daily.

Neurological examination at that time was normal, as was contrast-enhanced cranial MRI and haematological analysis. Indomethacin given at a dose of $200 \mathrm{mg}$ /day resulted in complete cessation of the attacks within $48 \mathrm{~h}$. After reduction of indomethacin to $100 \mathrm{mg}$ per day for 3 weeks no attacks recurred, but a mild retroorbital pain reappeared. In the following weeks the indomethacin dosage was further tapered in a stepwise fashion. The overall indomethacin treatment period lasted 6 months. At final follow-up examination 4 months later the patient was still without any medication and headache free.

\section{Case 2}

A 38-year-old nurse had a history of migraine with aura in childhood, neurodermatitis, immunodeficiency with depressed T4 lymphocytes and genital HPV infection. She presented with a progressing decrease of temporal vision over her right eye without headache or diplopia. On the otherwise normal examination, visual acuity O.D. was $1 / 10$ s.c. and there was a mild temporal visual field defect in the right eye. During right eye pressure she reported retroorbital pain. Analysis of the CSF revealed a minor lymphocytic pleocytosis (cells $19 / \mathrm{mm}^{3}$ ) with normal CSF protein and no oligoclonal bands or elevated IgG. Cranial Gadolinium enhanced MRI showed focal contrast enhancement of the right optic nerve distal to the optic canal (Fig. 1A). Two weeks later the retroorbital pain and monocular scotoma had increased. Clinically a right afferent pupillary defect was found; visual acuity O.D. was $1 / 40$ s.c. Visual evoked potentials could not be elicited on the right side and showed increased latencies in the clinically asymptomatic left eye (P100:112.8 ms). Haematological studies showed positive antinuclear antibodies $(1: 240)$, positive antibodies against smooth muscle and slightly elevated cardiolipin $\operatorname{IgM}$ antibodies (13.9 MPL-U/ml). The other parameters were normal (ESR, immunoelectrophoresis, cardiolipin IgG antibodies, C3, C4, ANCA, AMA, blood glucose). Treatment with oral methylprednisolone 80 mg daily improved visual acuity (O.D. 1/10 s.c.). Cranial MRI was now normal.

The steroids were tapered twice, but right retroorbital pain recurred at doses of $20 \mathrm{mg}$ with an intensity of 7 on a scale of 10 . At this time she had complete blindness of the right eye with no afferent pupillary response. The second time the patient reported unbearable knife-like pain attacks (intensity 10/10), right temporo-orbital occurring 20-30 times a day and lasting about 5 to $10 \mathrm{~min}$. Increasing methylprednisolone dosage to $80 \mathrm{mg}$ daily in combination with indomethacin (up to $200 \mathrm{mg}$ daily as needed) almost completely abolished both kinds of pain. After 2 days' treatment with $80 \mathrm{mg}$ methylprednisolone only mild (intensity $2 / 10$ ) and rare attacks of right temporo-orbital headache occurred lasting only a few minutes and which completely responded to low doses of indomethacin $(50 \mathrm{mg})$. There was an incomplete recovery of vision in the right eye with perception of light and movement. At that time cranial Gadolinium-enhanced MRI showed a small contrast-enhancing mass of 3-4 mm under the right optic nerve between the optic canal and the chiasm and an asymmetry of the cavernous sinus with enlargement of the right side due to intracavernous contrast-enhancing soft tissue lateral to the internal carotid artery (Fig. 1B). In a follow-up study 6 weeks later this space-occupying lesion was reduced in size (Fig. 1C). Indomethacin was stopped after an overall treatment period of 7 weeks. After 12 months there was no more space occupying lesion. The patient was free of headache and the visual acuity was 0.1 s.c. on the right. Steroids were tapered very slowly and stopped after a 16 months' treatment course.

\section{Discussion}

In both patients accurate diagnosis according to the IHS criteria is not possible. Only the first headache episode in patient 1 showed the typical features of THS, but the involvement of the second branch of the trigeminal nerve is uncommon. Patient 2 presented with a "Tolosa-Hunt-like" syndrome, since she presented with an isolated involvement of the optic nerve without paralysis of one of the oculomotor nerves. Optic neuritis might present with a similar clinical picture and therefore is the main differential diagnosis, but MRI scans demonstrated a space-occupying lesion in and near the cavernous sinus which corresponded to the radiological findings in THS as described in the literature (3). This lesion promptly responded to steroids.

Some weeks later, both patients developed a second distinct headache syndrome which had most typical features of $\mathrm{CPH}$ with regard to the nature of the pain, the frequency and duration of attacks and remission of pain only after administration of high doses of indomethacin, but without autonomic features.

Transitions from one type of headache to another and overlap syndromes have been reported for migraine to cluster headache $(\mathrm{CH})(4,5)$, THS $(6)$, and $\mathrm{CPH}(7), \mathrm{CH}$ to THS (6), THS to $\mathrm{CH}(6)$, and $\mathrm{CH}$ to $\mathrm{CPH}(8)$. Although recurrent attacks of unilateral stabbing pain between the episodes have been described in $27 \%$ of cases with recurrent THS (4), their clinical features remain unclear. To our knowledge these are the first cases in the literature with 

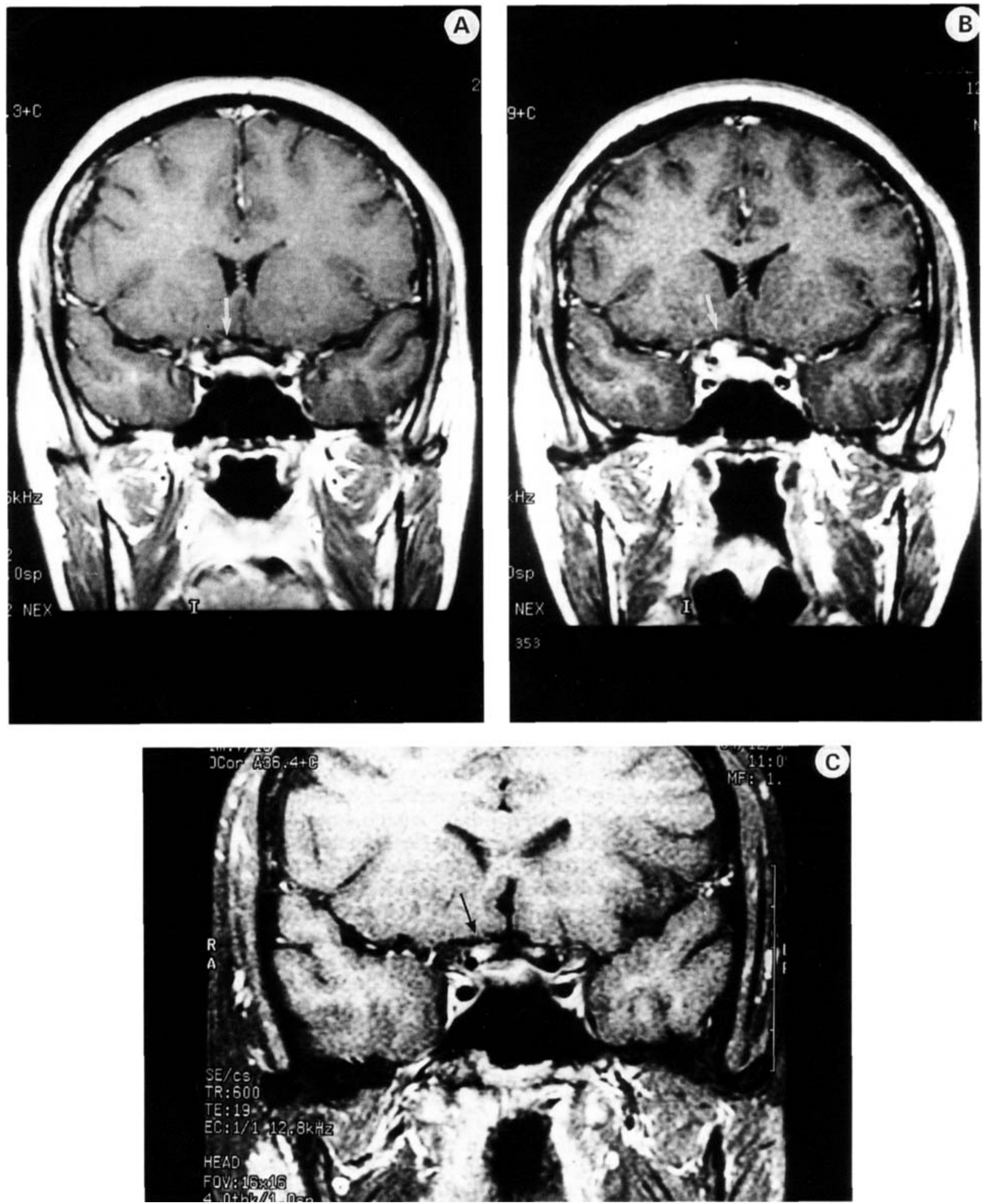

Fig. 1. A. Contrast enhanced MRI of patient 2. Frontal, T1-weighted scan with focal contrast enhancement of the right optic nerve. B. Frontal, T1-weighted scan (contrast enhanced) shows a small contrast-enhancing mass under the right optic nerve and an enlargement of the right cavernous sinus. C. Frontal T1-weighted scan (contrast enhanced) shows a reduction in size of the small space-occupying contrast-enhancing lesion in the region of the right optic nerve on follow up. 
"Tolosa-Hunt-like" syndromes that preceded another orbital pain syndrome resembling $\mathrm{CPH}$ without autonomic features. The histopathology in patients with THS, as well as the findings on orbital phlebography, suggests that the cause of THS is a nonspecific granulomatous inflammation with venous vasculitis in the orbital and sinus cavernosus regions (9). In patients with $\mathrm{CPH}$, Antonaci (10) found abnormalities in orbital phlebography in three out of five patients. The MRI findings in one of our patients agreed with the corresponding radiological pathologies in the cavernous sinus reported by Yousem et al. in 9 of 11 patients with THS (confirmed on biopsy in three cases) (3).

The transition of a THS-like pain in our patients supports the concept that $\mathrm{CPH}$ might also be caused by inflammation in the region of the sinus cavernosus. Orbital phlebography in patients with $\mathrm{CPH}$ has revealed signs of venous vasculitis $(10,11)$ with similar findings to those reported in THS (12), other unilateral headache syndromes like $\mathrm{CH}$ (13) and SUNCT (shortlasting unilateral neuralgiform headache with conjunctival injection and tearing) (14). There are also reports of patients with periorbital pain resembling that of THS and visual loss without ophthalmoplegia (15), as was described here in case 2. How inflammation in the region of the orbits and the cavernous sinus causes such different clinical syndromes as $\mathrm{CPH}, \mathrm{CH}$, THS, or SUNCT remains to be determined. We suggest that topographic differences in inflammatory sites, e.g. orbits/anterior cavernous sinus or posterior cavernous sinus, may be responsible for the different clinical features. In this sense, as suggested by Hannerz et al. 1986 (15), THS may be only one manifestation of an inflammatory process in the region of the anterior cavernous sinus (Fig. 1). Chronic paroxymal hemicrania may be another manifestation of such an inflammation with a different location. The varying responses to steroids and drugs such as indomethacin are probably due to differences in the underlying inflammatory mecha- nisms or the differential effects of venous pressure in the cavernous sinus.

Acknowledgements.-The authors thank Mrs M. Seiche and Mrs Benson for their help in editing the manuscript.

\section{References}

1. Sjaastad O, Dale I. Evidence for a new (?) treatable headache entity. Headache 1974;14:105-8

2. Classification and diagnostic criteria for headache disorders, cranial neuralgias and facial pain. Cephalalgia 1988;8 Suppl 7:1-96

3. Yousem DM, Atlas SW, Grossman RI, Sergott RC, Savino PJ, Bosley TM. MR imaging of Tolosa-Hunt syndrome. AJNR 1990;10:1181-4

4. Sjaastad O. Cluster headache. In: Rose FC, editor. Handbook of clinical neurology, vol. 4 (48). Headache. Amsterdam: Elsevier, 1986a:217-46

5. Andersson PG. Migraine in patients with cluster headache. Cephalalgia 1985;5:11-16

6. Hannerz J. Recurrent Tolosa-Hunt syndrome. Cephalalgia 1992;12:45-51

7. Antonaci F, Sjaastad O. Chronic paroxysmal hemicrania (CPH): A review of the clinical manifestations. Headache $1989 ; 29: 648-56$

8. Tehindrazanarivelo AD, Visy YM, Bousser MG. Ipsilateral cluster headache and chronic paroxysmal hemicrania: two case reports. Cephalalgia 1992;12(5):318-20

9. Hannerz J, Ericson K, Bergstrand G. Orbital phlebography in patients with Tolosa-Hunt syndrome in comparison with normal subjects. Acta Radiol (Diagn) 1984;25:457-63

10. Antonaci $\mathrm{F}$. Chronic paroxysmal hemicrania and hemicrania continua: orbital phlebography and MRI studies. Headache 1994;34:32-4

11. Hannerz J, Ericson K, Bergstrand G. Chronic paroxysmal hemicrania: orbital phlebography and steroid treatment. $\mathrm{Ce}^{-}$ phalalgia 1987;7:189-92

12. Muhletaler C, Gerlock AJ. Orbital venography in painful ophthalmoplegia (Tolosa-Hunt syndrome). Am J Roentgenol 1979;34:31-4

13. Hannerz J, Erikson K, Bergstrand G. Orbital phlebography in patients with cluster headache. Cephalalgia 1987;7:207-11

14. Kruszewski P. Shortlasting, unilateral, neuralgiform headache attacks with conjunctival injection and tearing (SUNCT syndrome): orbital phlebography. Cephalalgia 1992;12:387-9

15. Hannerz J, Ericson K, Bergstrand G. A new etiology for visual impairment and chronic headache. Cephalalgia 1986;6:59-63 